\title{
ReaR
}

\section{Estabilidad de los equipos de trabajo: buscando la mejora en el rendimiento quirúrgico}

Artículo original: Implications of Perioperative Team Setups for Operating Room Management DecisionsDietrich Doll, MD, PhD, Peter Kauf, PhD, Katharina Wieferich, BASc, Ralf Schiffer, BHA, and Markus M. Luedi, MD, MBA Anesth Analg 2017; 124:262-9 (․ML)

Muñoz Expósito R, Muñoz Corsini L.

Hospital Universitario de Guadalajara.

\section{Resumen}

Conseguir el máximo rendimiento de las jornadas quirúrgicas es objetivo de gestión asistencial y motivo de continuo estudio en sus diferentes variables incluyendo la actuación en equipo en el entorno perioperatorio; pero el impacto administrativo de grupos estables de anestesiólogos y cirujanos no está claro. La hipótesis del presente estudio es que la eficiencia de la jornada mejora con grupos estables de anestesistas y cirujanos. Evalúan 13.632 cirugías en las que asignan diferentes anestesistas a cirujanos específicos (ajustando los datos según el tipo de paciente y la especialidad quirúrgica) y analizan los tiempos de recambio entre cirugías. La asignación del anestesiólogo más adecuado en una lista quirúrgica y a un cirujano en concreto disminuyó en un 6,8\% los tiempos medios de recambio (IC 95\%) mejorando la eficiencia en el bloque quirúrgico.

\section{Introducción}

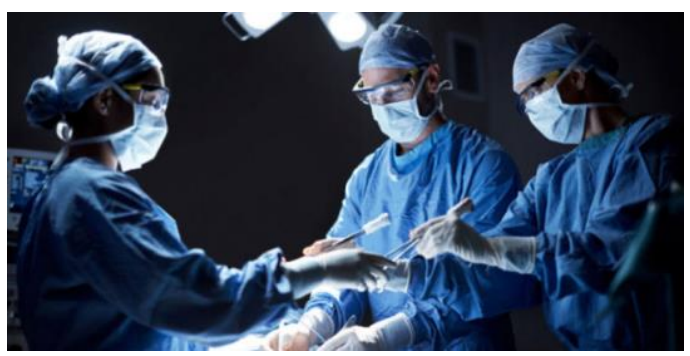

Conseguir el máximo rendimiento de las jornadas quirúrgicas es objetivo de la gestión asistencial y un motivo de continuo estudio en sus diferentes variables incluyendo la actuación en equipo en el entorno perioperatorio; pero el impacto administrativo de grupos estables de anestesiólogos y cirujanos no está claro. La hipótesis del presente estudio se basa en que la eficiencia de la jornada mejora con grupos estables de anestesistas $y$ cirujanos. Evalúan 13.632 cirugías en las que destinan diferentes anestesiólogos a cirujanos específicos (ajustando los datos según el tipo de paciente y la especialidad quirúrgica) y analizan los tiempos de recambio entre cirugías. La asignación del anestesiólogo más adecuado en una lista quirúrgica y a un cirujano en concreto reduce en un $6,8 \%$ los tiempos medios de recambio (IC 95\%) mejorando la eficiencia en el bloque quirúrgico.

La sobreutilización del tiempo quirúrgico (cuando los quirófanos destinados a una especialidad concreta necesitan para concluir más tiempo del previsto) y los tiempos de recambio entre pacientes (periodo desde el fin del procedimiento por parte del cirujano y las actividades relacionadas con el paciente hasta el momento de inicio del siguiente procedimiento) son unas medidas de eficiencia quirúrgica bien establecidas (1) (2) (3). 
Está comprobado que una adecuada comunicación en el equipo mediante la implementación de un listado de verificación quirúrgica mejora la seguridad del paciente y la eficiencia del proceso. Por el contrario, la actuación de una plantilla quirúrgica con una composición inconsistente está asociada a un tiempo quirúrgico mayor, una estancia hospitalaria prolongada y tasa de reingresos a los 30 días más elevada ${ }^{(4)}$.

Algunos estudios han visto cómo la combinación específica del personal puede mejorar la eficiencia en cirugía de mano o en cirugía mínimamente invasiva (5). A día de hoy, solo una publicación incluye tanto a anestesiólogos como a cirujanos para intentar minimizar las tareas no relacionadas con la propia intervención, pero no analiza la interacción dentro del conjunto (6). El presente artículo pretende explorar las implicaciones derivadas de las diferentes combinaciones del personal quirúrgico a la hora de tomar decisiones y si estas redundarán en menores tiempos de recambio entre pacientes $y$ mayor eficiencia.

\section{Descripción del artículo}

Se trata de un estudio retrospectivo llevado a cabo en un hospital alemán de 321 camas durante 71 meses (mayo 2007 hasta abril 2013) recogiendo un total de 36.834 casos, analizados de forma anónima. Durante este periodo no hubo ningún tipo de cambio en los protocolos de quirófano ni en el equipo directivo.

Un total de 5 quirófanos estaban operativos de 8:00 a 15:30 (7,5 horas) los días laborables con una media de 4 intervenciones quirúrgicas (en un intervalo de 1 a 15 cirugías). Los anestesiólogos no estaban subespecializados en cirugías concretas, sino que cubrían todas las especialidades de forma rotatoria. Participaron anónimamente 64 cirujanos (asignados con $\mathrm{S}$ y un número) y 48 anestesiólogos (asignados con A y un número).

Para asegurar unas condiciones comparables (que todos los casos estudiados estuvieran precedidos de una intervención previa), fueron excluidos los primeros procedimientos quirúrgicos de cada día, los cambios de última hora y aquellas cirugías de duración superior a 90 minutos quedando finalmente 13.632 procedimientos quirúrgicos analizables.

Se realizó un análisis multivariable, para cuantificar los efectos de probables factores de confusión en variables como la edad, la categoría $A S A$ (American Society of Anesthesiologists), la lista quirúrgica, la duración del procedimiento quirúrgico actual y la del previo. Otras covariables tenidas en cuenta para minimizar los factores de confusión fueron el procedimiento realizado, el género, índice de masa corporal y las comorbilidades. Se asumió una diferencia estadísticamente significativa si $\mathrm{p}<0,05$. Todos los test fueron considerados a dos colas (bilaterales), es decir, se compararon dos muestras para saber si diferían entre sí, sin preguntarse cuál de ellas tenía mayor valor estadístico; considerando ambos lados de la media de una curva de distribución.

En los datos pareados, se utilizó la correlación de Spearman para una distribución no normal. Para todas las comparaciones de las tendencias centrales, se usó el test de Wilcoxon y en la comparación de más de dos grupos, el test de Kruskal Wallis.

Para la corrección de errores en los test múltiples, el valor de $\mathrm{p}$ se ajustó según el método Holm para intentar 
evitar el error tipo I. Se asignaron letras por grupos, de forma que los grupos con diferencias estadísticamente significativas no compartían letras.

Para evaluar el aprendizaje individual y en equipo, los tiempos de recambio ajustados por cada individuo y cada equipo fueron correlacionados con sus respectivos números de casos usando la correlación de Spearman. Sólo los individuos y equipos que realizaron más de 2 procedimientos fueron tenidos en cuenta en este análisis.

\section{Algoritmo de decisión para procedimientos quirúrgicos}

Los investigadores aplicaron un método conocido como simulación de Montecarlo en el que se obtuvieron combinaciones aleatorias para simular diferentes variables. Con el objetivo de evitar sesgos de análisis sobre las diferencias en los tiempos de recambio, sólo se eligieron cirujanos con más de 20 casos y anestesiólogos que realizaron al menos 13 procedimientos.

Como resultado, se obtuvieron tablas de letras indicando las diferencias significativas por anestesiólogo para cada cirujano, simulando asignaciones más efectivas de acuerdo con los siguientes pasos:

1. Se escogieron los casos quirúrgicos en los que participaron cirujanos con al menos 20 casos, por lo que quedaron 13.447 procedimientos de los 13.632 originales.

2. Se crearon las tablas de decisión para los 13.447 procedimientos quirúrgicos.

3. Se seleccionaron al azar las 13.447 combinaciones de profesionales.

A cada cirujano en concreto, se le designó al azar un anestesiólogo con el que había trabajado al menos una vez (según las tablas del punto 2). Esos tiempos de recambio para cada uno de los 13.447 casos se agregaron posteriormente para obtener una suma total de tiempos de recambio.

- Asignación mejorada del anestesiólogo: si no había diferencias significativas entre los anestesistas asignados al cirujano, se adjudicaba un nuevo anestesiólogo al azar. Si había diferencias significativas, se calculaba el tiempo medio de recambio ajustado con el mejor rendimiento entre el grupo de anestesiólogos para ese cirujano y se sumaba para obtener un cómputo total de tiempos de recambio mejorados.

- Se realizaron 200 interacciones de los pasos 3 al 5; en total 200 muestras de la simulación de Monte Carlo.

\section{Resultados obtenidos}

Demográficos: La media de edad fue de 48,44 años distribuida irregularmente en las listas quirúrgicas de forma significativa $(\mathrm{p}<0,0001)$ así como en los grupos de la clasificación $A S A$ : 2.530 fueron ASA I, 8.213 ASA II, 2.704 ASA III y 275 ASA IV.

Tiempos: La media de los tiempos de recambio entre pacientes fue de 39,2 minutos y la de los tiempos quirúrgicos de 57,9 (mínimo 1,2 y máximo 502,2 minutos).

Los datos mostraron preferencias en la asignación de ciertos anestesiólogos a ciertas listas de especialidades quirúrgicas $(\mathrm{p}<0,001)$.

Efectos individuales en el tiempo de recambio: 
Dependieron significativamente del cirujano, así como del anestesiólogo ( $\mathrm{p}$ $<0,0001)$.

Aprendizaje individual a lo largo del tiempo: Para cada especialista se tuvieron en cuenta los tiempos medios de recambio, y su correlación con el aumento progresivo de casos. Para 5 cirujanos, se apreció una correlación significativa (después del ajuste de Holm, $\mathrm{p}<0,0002$ ) y para 3 de ellos una correlación negativa. Para los anestesiólogos, no se encontraron diferencias estadísticamente significativas en el aprendizaje en relación con el número de casos.

Aprendizaje en equipo: De las 3.072 posibles plantillas quirúrgicas (combinando 64 cirujanos y 48 anestesiólogos) se hallaron 853 agrupaciones con al menos un procedimiento y 659 con al menos dos intervenciones quirúrgicas. No se apreció correlación significativa entre el número de casos y los tiempos de recambio de los grupos. El aprendizaje a lo largo del tiempo fue despreciable en el conjunto a estudio, lo que quiere decir que la misma pareja de cirujanoanestesiólogo no mejoró a lo largo del tiempo a pesar del aumento en el número de intervenciones juntos.

Actuación individual y en equipo en las listas quirúrgicas estudiadas: En cuanto al tiempo de recambio, se encontraron diferencias significativas entre los cirujanos $(p<0,0001)$ en todos sus casos, sin tener en cuenta al anestesiólogo asignado. Éstos, independientemente del cirujano establecido, también mostraron diferencias significativas $(\mathrm{p}<0,0001)$.

Desglosando por especialidades quirúrgicas; aunque hubo diferencias en la actuación entre los anestesiólogos en otorrinolaringología, cirugía general y ginecología; no se vieron diferencias significativas en traumatología.

Rendimiento plantilla - dependiente. Algoritmo y evaluación: El algoritmo definido pretendió reducir los tiempos de rotación entre pacientes.

Paso 1: Comprobar si el cirujano tenía un rendimiento cambiante con los diversos anestesiólogos usando las tablas creadas. Si existían diferencias, ir al paso 2. Si no, asignar a cualquiera de los anestesiólogos e ir al paso 3.

Paso 2: Comprobar qué grupo de anestesiólogos mostró la mejor actuación con el cirujano dado, asignar un anestesiólogo fuera de ese grupo.

Paso 3: Hacer lo mismo con el siguiente cirujano.

Todas las listas quirúrgicas, salvo en traumatología, mejoraron así como también los tiempos de recambio en general. Estos últimos se optimizaron en un $6,8 \%$ con un tiempo medio de reemplazo entre pacientes de 39,2 minutos (IQR, 28,8-46,2).

\section{Discusión y análisis crítico del artículo}

De forma general, la hipótesis del estudio está claramente definida: ver si las diferentes combinaciones de anestesiólogos y cirujanos implican menores tiempos de reposición entre pacientes y si esos equipos que trabajan juntos a lo largo del tiempo llegan a ser más eficientes.

Analizando la población a estudio, se estandarizó a los pacientes, intentando eliminar factores de confusión como la edad, ASA o el procedimiento al que iban a ser sometidos.

Con respecto a la validez de los resultados obtenidos, se observa una 
mejoría en la eficiencia al aplicar el algoritmo. Nos encontramos con intervalos de confianza aceptables $(95 \%)$ y con variables objetivas para analizar los datos obtenidos. Los resultados son creíbles pero los factores que pueden influir en ellos son tantísimos que muchos no se han tenido en cuenta y podrían ser importantes, tales como la personalidad de cada componente del equipo, el resto del personal que trabaja en el bloque quirúrgico, la idoneidad de las instalaciones, el circuito físico que siguen los pacientes al entrar y salir de quirófano o restricciones de tiempo.

La asignación del correcto anestesiólogo a una lista quirúrgica y a un cirujano en concreto puede afectar a la actuación del equipo, a la sobreutilización del tiempo quirúrgico y a la eficiencia (1) (2) (6). La disponibilidad de herramientas informáticas para identificar configuraciones óptimas podría reducir los tiempos de sobreutilización y por tanto tener una implicación administrativa positiva. Elegir los miembros adecuados de un grupo es vital para promover equipos de alto rendimiento basados en el liderazgo ${ }^{(2)}$.

El algoritmo de decisión no considera algunas limitaciones, como los recursos que tiene un determinado anestesiólogo en un momento determinado, centrándose en obtener la actuación más eficiente en cada momento. También omite las preferencias de los anestesiólogos a la hora de trabajar en una especialidad en concreto.

El manejo de un quirófano no se puede basar completamente en ecuaciones matemáticas y algoritmos. Se debe tener en cuenta factores como el temperamento, los gustos, la percepción individual, la forma de trabajar, etc ${ }^{(3)}$.
Los tiempos de rotación dependen de complejas interacciones que obedecen a múltiples factores adicionales como la preparación de los instrumentos quirúrgicos, la disponibilidad del personal, el circuito de pacientes, la hora del día o las tareas de limpieza ${ }^{(3)}$. En el estudio los tiempos de recambio resultantes fueron obtenidos de una selección al azar, construyendo medias de equipo cirujano-anestesiólogo que pueden diferir de las reales. No se ha tenido en cuenta al resto del personal que trabaja en el bloque quirúrgico (enfermería, auxiliares, celadores, equipo de limpieza...) y como la interacción entre todos puede mejorar o empeorar los tiempos de actuación ${ }^{(5)}$.

Con respecto a la validez externa del estudio, el hospital podría parecerse en tamaño al Hospital Universitario de Guadalajara (321 camas vs 410). Bien es cierto que el hospital alemán donde se realizó el estudio no cuenta con residentes, que también podrían influir tanto positiva como negativamente en los tiempos de recambio. La asignación del equipo quirúrgico y el algoritmo de actuación se ha visto que funcionan en este hospital en concreto, pero debería extrapolarse a otros entornos y ver si es igualmente válido en otros hospitales.

En grandes centros es muy complicado organizar las diferentes combinaciones del tándem anestesiólogo-cirujano, porque pueden llegar a ser infinitas o necesitar mucho tiempo para llegar a analizar la efectividad de todas ellas. Al tener muchos grupos con potencial de interacción, los test múltiples podrían ser cuestionables para identificar una actuación más lenta o rápida.

Uno de los resultados más sorprendentes que nos ha mostrado este estudio es que ni individuos ni equipos quirúrgicos adquieren un aprendizaje basado en el número de procedimientos realizados. Las discusiones abiertas 
sobre el trabajo conjunto son la clave para crear una cultura de seguridad (1) (2). Al realizar asignaciones al azar, no se tiene en cuenta ninguna estrategia externa que fomente el trabajo en grupo.

El manejo de quirófano, administrativamente hablando, y los tiempos de recambio entre pacientes dependen de muchos factores, y las interacciones humanas se encuentran en la raíz de la mayoría de las variables. Optimizando esas interacciones, asociando un cirujano con el anestesiólogo con el que es más eficiente, se pueden reducir esos tiempos de reemplazo con las implicaciones administrativas que ello podría tener para disminuir las listas de espera quirúrgicas. Otras estrategias de asignación anestesiólogo-cirujano podrían ser interesantes para nuevos estudios futuros.

\section{Conclusiones}

* La seguridad, la calidad y la eficiencia en la interacción de un grupo multidisciplinar quirúrgico es de vital importancia a la hora de estudiar los tiempos quirúrgicos $\mathrm{y}$ de rotación entre pacientes.

*A través de un algoritmo de actuación informatizado según las diferentes combinaciones de especialistas es más fácil obtener la ecuación más eficiente para cada procedimiento y mantener así una buena productividad, si bien es cierto que este modelo es idílico y difícil de aplicar en la práctica diaria.

*En los tiempos de recambio no sólo influye la combinación cirujanoanestesiólogo, sino la del resto del personal y otros factores difíciles de cuantificar.

*En este estudio se ha visto que no hay un aprendizaje y mejora de los tiempos de rotación a pesar de que aumente el número de procedimientos que hagan juntos el mismo cirujano y anestesiólogo. Por ello, sería útil investigar qué factores influyen en esa falta de aprendizaje y potenciar el trabajo en equipo (por ejemplo, a través de simulaciones, del listado de verificación quirúrgica segura (LVS) de la Organización Mundial de la Salud, realizando debriefings de lo aprendido...).

\section{Bibliografía}

1. Gómez-Ríos MA, Abad-Gurumeta A, Casans-Francés R, Calvo-Vecino JM. Claves para optimizar la eficiencia de un bloque quirúrgico. Rev Esp Anestesiol Reanim. 2018. Redar-962 https://doi.org/10.1016/j.redar.2018.08. $\underline{002}$

2. Gómez-Arnau JI, González A. Principios generales de organización y gestión clínica de un bloque quirúrgico. Rev. Esp. Anestesiol. Reanim. 2001; 48: 180-187.

3. Doll D, Wieferich K, Erhart T, Hoenemann C. Waiting for Godot: An analysis of 2622 operating room turnover times. Eur $J$ Anaesthesiol. 2014;31:388-389. (ubMed)

4. Xiao Y, Jones A, Zhang BB, et al. Team consistency and occurrences of prolonged operative time, prolonged hospital stay, and hospital readmission: a retrospective analysis. World J Surg.

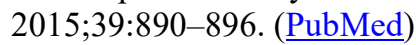

5. Avery DM III, Matullo KS. The efficiency of a dedicated staff on operating room turnover time in hand surgery. $J$ Hand Surg Am. 2014;39:108-110. ( PubMed)

6. Harders M, Malangoni MA, Weight S, Sidhu T. Improving operating room efficiency through process redesign. Surgery. 2006;140:509-514. (PubMed) 
Correspondencia al autor

\section{Raquel Muñoz Expósito}

raquelmuex@gmail.com

Residente Anestesiología y Reanimación.

Lourdes Muñoz Corsini
Imunoz.corsini@gmail.com

FEA Anestesiología y Reanimación.

Hospital Universitario de Guadalajara.

Aceptado para el blog en mayo de 2019 